Proceedings of XIX International Scientific Conference "New Technologies and Achievements in Metallurgy, Material Engineering, Production Engineering and Physics", Częstochowa, Poland, June 7-8, 2018

\title{
Magnetocaloric Effect in Annealed (Mn,W)-Co-Ge Alloy
}

\author{
P. GęBara* And K. Kutynia
}

Institute of Physics, Faculty of Production Engineering and Materials Science, Częstochowa University of Technology, al. Armii Krajowej 19, 42-200 Częstochowa, Poland

The aim of the present work was to study the influence of partial substitution of $\mathrm{Mn}$ by $\mathrm{W}$ in an $(\mathrm{Mn}, \mathrm{W})-\mathrm{Co}-\mathrm{Ge}$ alloy. The master alloys were prepared by arc-melting a high purity of constituent elements under a low pressure of Ar. The obtained specimens were annealed at $1123 \mathrm{~K}$ for 5 days in sealed quartz tubes under the low pressure of a protective Ar gas. The magnetic properties of the prepared samples were measured at a wide range of temperatures by a Quantum Design MPMS-XL 5 equipped with a $5 \mathrm{~T}$ superconducting magnet.

DOI: 10.12693 /APhysPolA.135.298

PACS/topics: $75.30 . \mathrm{Sg}, 75.50 . \mathrm{Bb}$

\section{Introduction}

The magnetocaloric effect (MCE) is observed in magnetic materials exposed to the variation of an external magnetic field [1]. Such a phenomenon is manifested as a temperature change in the magnetic material. For over two decades, different materials exhibiting the MCE have been investigated. The most common MCE materials are pure Gd [2] and its alloys [3, 4], the $\mathrm{La}(\mathrm{Fe}, \mathrm{Si})_{13}$-type alloys [5, 6] or manganites [7]. Recently, the full Heusler [8] and semi-Heusler alloys [9] have been intensively studied. $\mathrm{Li}$ and co-workers in [10] measured relatively high values of magnetic entropy change, which reached $15 \mathrm{~J} /(\mathrm{kg} \mathrm{K})$ under the change of an external magnetic field of $2 \mathrm{~T}$. The magnetic moment of tungsten is $2 \mu_{\mathrm{B}}$ and such an element could be used as a substitution of $\mathrm{Mn}$ in the $\mathrm{MnCoGe}$ structure. Moreover, the ionic radius of $\mathrm{W}$ is slightly lower than $\mathrm{Mn}$, which could have an effect on the structure and magnetic properties of $\mathrm{MnCoGe}$ alloy. Such an investigation has not been carried out previously. Accordingly, the aim of the present work was to study the influence of the substitution of $\mathrm{Mn}$ by $\mathrm{W}$ in the MnCoGe alloy.

\section{Sample preparation and experimental details}

The sample with a nominal composition of $\mathrm{Mn}_{0.9} \mathrm{~W}_{0.1}$ CoGe was prepared by arc-melting high purity elements under the protective atmosphere of $\mathrm{Ar}$ gas. The sample was remelted several times in order to ensure homogeneity. The XRD studies were carried out using a Bruker D8 Advance diffractometer with $\mathrm{Cu} K_{\alpha}$ radiation and a semiconductor detector, LynxEye. The $\mathrm{X}$-ray pattern was analyzed using Bruker EVA software. The magnetic studies (the Curie temperature and magnetocaloric effect) were conducted using a Quantum

*corresponding author; e-mail: pgebara@wip.pcz.pl
Design Physical Properties Measuring System (PPMS) model 6000 equipped for working with a wide range of magnetic fields and temperatures.

\section{Results and discussion}

The XRD pattern of the investigated alloy is depicted in Fig. 1. The analysis revealed a coexistence of two orthorhombic phases with different lattice parameters. An occurrence of orthorhombic phases suggests that material is in a paramagnetic state. The lattice parameters of the recognized phases were collected in Table I.

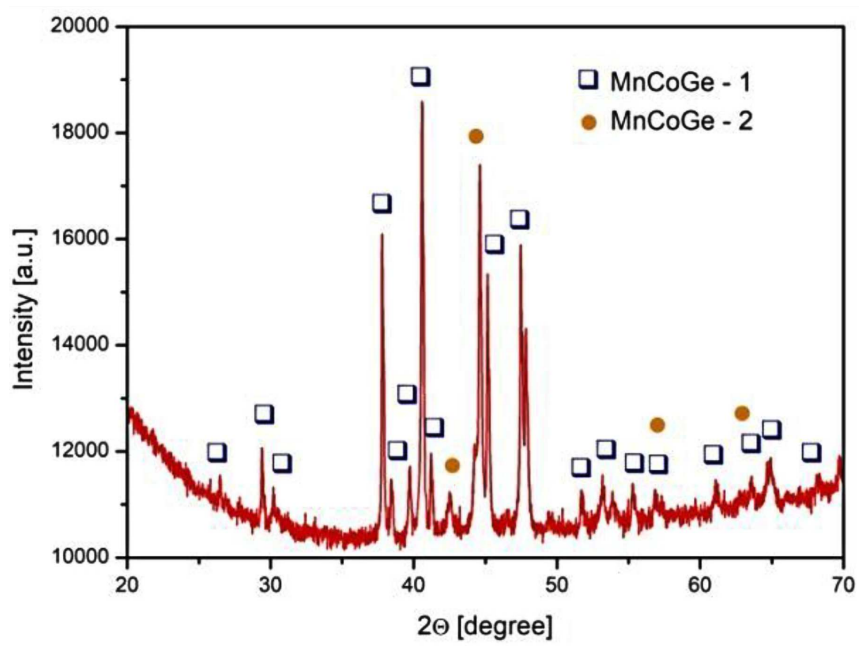

Fig. 1. The XRD pattern of the studied alloy.

TABLE I

Lattice parameters of phases detected in the investigated alloy.

\begin{tabular}{c|c|c|c}
\hline \hline \multirow{2}{*}{ Phase } & \multicolumn{3}{|c}{ Lattice parameter } \\
\cline { 2 - 4 } & $a[\AA]$ & $b[\AA]$ & $c[\AA]$ \\
\hline MnCoGe-1 & 5.947 & 3.826 & 7.051 \\
MnCoGe-2 & 5.316 & 4.087 & 7.079
\end{tabular}




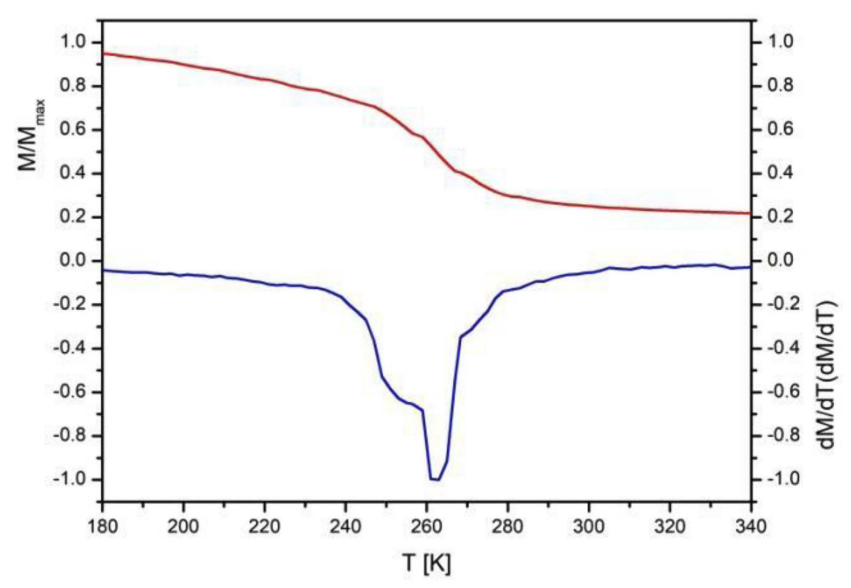

Fig. 2. Temperature dependence of magnetization (up) and its first derivative (down) collected for the studied sample.

Two different orthorhombic phases (both with space group Pnma) indicates a random distribution of elements in the structure. Moreover, it is impossible to recognize correctly, which phase consists of tungsten. The existence of the two different phases with a different lattice constant suggests that a temperature dependence of magnetization should reveal two Curie temperatures in this material. The $M=f(T)$ curve is plotted in Fig. 2 .

The analysis of the temperature dependence of magnetization revealed two Curie temperatures, 252 and $264 \mathrm{~K}$. Determining the values of the Curie temperature confirmed previous predictions that the material is in a paramagnetic state.

In order to determine the magnetocaloric effect, the field dependences of magnetization collected in a wide temperature range were measured. Based on $M=$ $f(T, H)$ curves, a magnetic entropy change $\Delta S_{M}$ was calculated using the thermomagnetic Maxwell equation [11]:

$$
\Delta S_{M}(T, \Delta H)=\mu_{0} \int_{0}^{H}\left(\frac{\partial M(T, H)}{\partial T}\right)_{H} \mathrm{~d} H,
$$

where $\Delta S_{M}$ is the magnetic entropy change, $M$ is magnetization, $T$ is temperature, $H$ is the magnetic field and $\mu_{0}$ is the magnetic permeability of the vacuum.

The temperature dependences of the magnetic entropy change are shown in Fig. 3.

Two peaks are clearly seen in Fig. 3. The first peak is visible in the vicinity of $250 \mathrm{~K}$ and the second one at $266 \mathrm{~K}$. Such values correspond well with the Curie points revealed using the $M$ vs. $T$ curve. The maximum values of $\Delta S_{M}$ reaches $3.3 \mathrm{~J} /(\mathrm{kg} \mathrm{K})$ under the change of an external magnetic field $5 \mathrm{~T}$, which is almost five times lower comparable to the results published in [10]. A low value of the maximum magnetic entropy change is probably caused by second order phase transition.

Such a shape of the $\Delta S_{M}$ vs. $T$ curve is expected due to the potential practical application of the produced alloy as an active element in magnetic refrigerators.

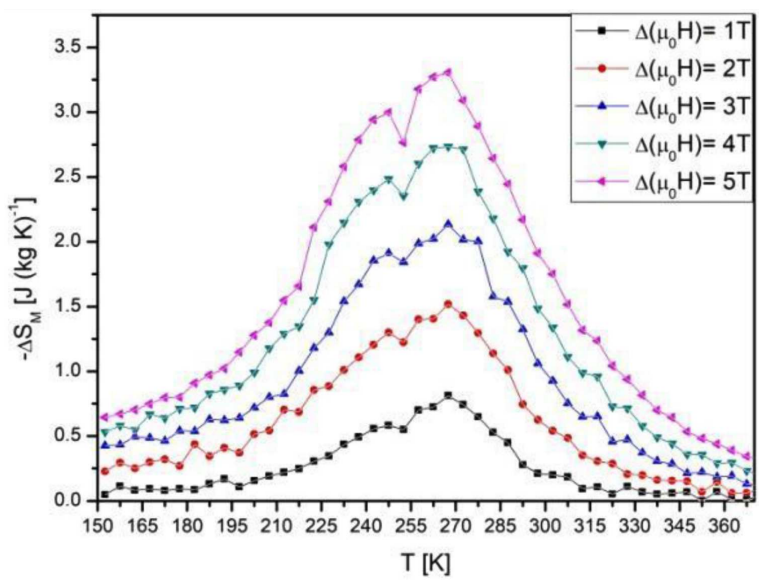

Fig. 3. $\Delta S_{M}$ vs. $T$ curves measured in different external magnetic fields.

Broadening of the $\Delta S_{M}$ vs. $T$ curve improves the temperature working range of the material. Moreover, high values of full width at half maximum $\delta T_{\mathrm{FWHM}}$ of $\Delta S_{M}=f(T)$ generates a relatively large refrigeration capacity RC. The RC was calculated using the following relation [12]:

$$
\mathrm{RC}\left(\delta T, H_{\mathrm{MAX}}\right)=\int_{T_{\text {cold }}}^{T_{\text {hot }}} \Delta S_{M}\left(T, H_{\mathrm{MAX}}\right) \mathrm{d} T,
$$

where $\mathrm{RC}$ is refrigeration capacity, $\delta T=T_{\text {hot }}-T_{\text {cold }}$ is the temperature range of the thermodynamic cycle ( $\delta T$ corresponds to full width at half maximum of magnetic entropy change peak) and $H_{\mathrm{MAX}}$ is the maximum value of the external magnetic field.

All thermomagnetic data were collected in Table II.

TABLE II

Thermomagnetic parameters revealed for investigated alloy.

\begin{tabular}{c|c|c|c}
\hline \hline $\begin{array}{c}\Delta\left(\mu_{0} H\right) \\
{[\mathrm{T}]}\end{array}$ & $\begin{array}{c}\Delta S_{M} \\
{[\mathrm{~J} /(\mathrm{kg} \mathrm{K})]}\end{array}$ & $\begin{array}{c}\delta T_{\mathrm{FWHM}} \\
{[\mathrm{K}]}\end{array}$ & $\begin{array}{c}\mathrm{RC} \\
{[\mathrm{J} / \mathrm{kg}]}\end{array}$ \\
\hline 1 & 0.81 & 70 & 40 \\
2 & 1.52 & 75 & 87 \\
3 & 2.13 & 80 & 135 \\
4 & 2.73 & 85 & 186 \\
5 & 3.30 & 90 & 234
\end{tabular}

It is clearly seen in Fig. 3 and in Table II that the full width at half maximum of $\Delta S_{M}$ peak is relatively high in comparison with the one achieved for the $\mathrm{La}(\mathrm{Fe}, \mathrm{Co}, \mathrm{Si})_{13^{-}}$ based multicomposite [13]. Moreover, values of the RC are relatively close to those delivered in [13].

\section{Conclusions}

In the present paper, the structure and thermomagnetic properties of the $\mathrm{Mn}_{0.9} \mathrm{~W}_{0.1} \mathrm{CoGe}$ alloy were investigated. The XRD studies of the sample in as-cast 
state carried out at room temperature revealed two orthorhombic structures with different lattice constants. The temperature dependence of magnetization and its first derivative exhibited two Curie points corresponding to recognized phases. Moreover, two overlapping peaks, corresponding to the detected Curie points were measured in the temperature dependence of the magnetic entropy change. The relatively high full width at half maximum of magnetic entropy change peaking at $90 \mathrm{~K}$ was detected. Such a high value gives the potential practical application of the alloy as an active magnetic regenerator in magnetic refrigerators.

\section{Acknowledgments}

This work was supported by the Rector of Częstochowa University of Technology, Professor Norbert Sczygiol.

\section{References}

[1] A.M. Tishin, Handbook of Magnetic Materials, Vol. 12, Ed. K.H.J. Bushow, Amsterdam 1999, p. 395.

[2] K.A. Gschneider, V.K. Pecharsky, in: Science Technology and Applications III, Eds. R.G. Bautista, C.O. Bounds, T.W. Ellis, B.T. Killbourn, The Minerals, Metals and Material Society, 1997, p. 209.
[3] M. Yue, J. Zhang, H. Zeng, H. Chen, X.B. Liu, J. Appl. Phys. 99, 08Q104 (2006).

[4] N. Pierunek, Z. Śniadecki, J. Marcin, I. Skorvanek, B. Idzikowski, IEEE Trans. Magn. 50, 2506603 (2014).

[5] A. Fujita, Y. Akamatsu, K. Fukamichi, J. Appl. Phys. 85, 4756 (1999).

[6] P. Gębara, J. Kovac, J. Magn. Magn. Mater. 454, 298 (2018).

[7] W. Zhong, W. Cheng, W.P. Ding, N. Zhang, Y.W. Du, Q.J. Yan, Solid State Commun. 106, 55 (1998).

[8] A. He, V. Svitlyk, Y. Mozharivskyj, Inorg. Chem. 56, 2827 (2017).

[9] H. Zhang, Y.W. Li, E.K. Liu, K. Tao, M.L. Wu, Y.W. Wang, H.B. Zhou, Y.J. Xue, C. Cheng, T. Yan, K.W. Long, Y. Long, Mater. Des. 114, 531 (2017).

[10] Y.W. Li, H. Zhang, K. Tao, Y.X. Wang, M.L. Wu, Y. Long, Mater. Des. 114, 410 (2017).

[11] V.K. Pecharsky, K.A. Gschneidner Jr, J. Magn. Magn. Mater. 200, 44 (1999).

[12] M.E. Wood, W.H. Potter, Cryogenics 25, 667 (1985).

[13] P. Gebara, P. Pawlik, J. Magn. Magn. Mater. 442, 145 (2017). 